\title{
La producción del sujeto en la formación para la investigación ${ }^{1}$
}

\section{The production of the subject in research training}

DOI: https://doi.org/10.32870/dse.v0i12.253

\section{Cristina Palomar Verea*}

Resumen: Este trabajo es una reflexión en torno al problemático panorama actual de la formación para la investigación en México, realizada a partir del trabajo cotidiano con alumnos de posgrado en una universidad pública. Se plantea que dicha formación transcurre sin considerar las dificultades que enfrentan los aprendices del oficio de la investigación tanto por llegar a este nivel educativo sin las bases necesarias, como porque los programas de formación, por una parte, omiten estas dificultades y por otra, privilegian la figura del investigador en detrimento de su tarea. Se exploran estas cuestiones con una pequeña investigación centrada en el punto de vista de los alumnos y se presentan brevemente las conclusiones obtenidas. Palabras clave: sujeto, formación para la investigación, oficio de la investigación, dispositivo de formación, investigador ideal.

Abstract: This paper is an inquiry into the current and problematic situation of research training in Mexico, as obtained from daily work with graduate students at a public university. It argues that such training takes place disregarding many of the difficulties faced by apprentices of professional research both because they have reached this level of education without learning the necessary baseline skills and knowledge, and because training programs do not consider these difficulties, but rather privilege the figure of the researcher at the expense of their task. These issues are explored through a brief inquiry centered on the students' viewpoints with concise conclusions. Keywords: Subject, professional research training, research profession, research training program, ideal researcher.

I.

Parece haber un acuerdo generalizado en México respecto a la creciente necesidad y a la gran importancia de impulsar la formación de nuevos investigadores en las instituciones de educación superior (IES). Este acuerdo parece sostenerse en el vínculo imaginario que suele establecerse demasiado fácilmente entre el número de investigadores y un pujante desarrollo nacional. Se asume el supuesto de que a mayor número de investigadores, se está más cerca del progreso y del resto de las metas del iluminismo. No obstante, dicho vínculo no debería darse por sentado ya que hay diversos factores que en nuestro país lo hacen problemático, algunos de ellos relacionados tanto con los procesos de formación de los nuevos investigadores, como con las posibilidades que éstos tienen en el contexto nacional actual de insertarse laboralmente en los ámbitos dedicados a la investigación científica y, de esa manera, poder realmente contribuir al desarrollo nacional. Por otra parte, habría que comprobar la relevancia que realmente tiene el resultado de la investigación científica en las decisiones vinculadas con el desarrollo nacional.

${ }^{1}$ Este trabajo se presentó, en una versión mucho más breve, como ponencia en el Seminario "Formando investigadores. Problemas y desafíos", que tuvo lugar los días 25 a 28 de noviembre del 2013, en Guadalajara, México, organizado por la Universidad de Guadalajara y la Universidad de Tres de Febrero.

* Psicoanalista y doctora en ciencias sociales con especialidad en antropología social. Profesora investigadora titular en el Departamento de Estudios en Educación de la Universidad de Guadalajara. Investigadora nacional nivel II. Correo electrónico: crispalvertina@hotmail.com 
Otro supuesto asumido en relación con la consideración positiva del crecimiento de los programas de formación para la investigación en México es que por ese medio se cumple el objetivo de enseñar a hacer investigación científica de acuerdo con los principios y reglas fundamentales de la ciencia universal, y a los estándares internacionales derivados de ellos. En estos programas se genera el sujeto de la ciencia nacional, que "adviene" como resultado de los discursos y saberes implicados en los programas de formación científica. Lo que se formula como resultado esperado en diversos programas de formación para la investigación, ${ }^{1}$ es lograr recursos humanos "de alto nivel", "con nivel de excelencia", "altamente capacitados", "con altos niveles de calidad y excelencia", "altamente calificados" o "con alto sentido del logro". Esta manera de referirse al sujeto esperado en los mencionados programas deja ver dos cuestiones: por una parte, que los programas se centran en producir un tipo particular de individuos caracterizados por los rasgos señalados; $y$, por otra, que no es claro el significado que en cada caso encierran los calificativos utilizados, los cuales parecen derivarse de los retos que la economía nacional e internacional plantean a las instituciones de educación actuales en nuestro país, combinados con las presiones demográficas y sociales, las exigencias políticas, las angustias presupuestales y los cambios culturales y educativos. Latapí (2007) señala que, a dichas instituciones

\begin{abstract}
Se les exige calidad, se las obliga a modernizarse, a ser eficientes, a preparar los cuadros que requiere el mercado, a desarrollar una cultura empresarial, a innovar en sus métodos pedagógicos y en sus procesos de gestión, a evaluarse y acreditarse sobre bases sólidas; y se les propone la "sociedad del conocimiento" como el paradigma obligado del futuro: si el conocimiento es - y lo será cada vez más - el eje vertebrador de las economías globalizadas, corresponde a los sistemas educativos y sobre todo a las universidades generar, proveer y distribuir ese conocimiento indispensable (Latapí, 2007: s/p).
\end{abstract}

En ese contexto, los temas de la excelencia y la calidad de la educación cobran enorme importancia, ya que están en consonancia con los requerimientos de cualquier mercancía que pretenda competir en el mercado global (lo cual se asume que deberán hacer los investigadores "de calidad"). Sin embargo, Latapí observa que no hay definiciones claras de lo que se entiende por calidad, aunque es frecuente encontrar que se confunde con el aprendizaje de contenidos académicos y con buenas calificaciones, pero también con cierta idea de "éxito" en el mundo laboral; tampoco queda muy claro cómo la excelencia -adjetivo superlativo- resulta útil para comprender lo que se espera de la formación científica. De ahí la pregunta siguiente: ¿Cómo intervienen en el proceso formativo de los nuevos investigadores estas expectativas que se centran más en sus personas que en su educación como investigadores?

1 Para esta afirmación se revisaron los objetivos que aparecen en las páginas electrónicas de algunos de los posgrados que forman investigadores tanto en la UNAM, como en la UdeG, el CINVESTAV y CIESAS. Se revisaron los objetivos de los programas de las siguientes áreas: ciencias sociales, ciencias matemáticas, antropología social, comunicación, ciencias biomédicas y educación, en las IES mencionadas. 
Este trabajo es una reflexión en torno a este panorama problemático, nutrida además por las preocupaciones surgidas en el trabajo cotidiano con alumnos de posgrado en una universidad pública mexicana, y que ha dado lugar a las siguientes preguntas: ¿Cómo y por qué caminos se llega a ser investigador/a?, ¿cómo es la experiencia de formación?, ¿cómo se define el oficio de la investigación a partir de dicha experiencia?, ¿cómo son vistos los profesores y directores de tesis, en tanto actores centrales de la formación para la investigación, y qué tipo de relación establecen los alumnos con ellos?, ¿cuál es el tipo ideal del investigador que se produce en los programas de formación?

Se buscó respuesta a estas interrogantes con un estudio sencillo cuyo punto de partida fue la afirmación de que el sistema de posgrados en nuestro país tiene, además del objetivo explícito de formar investigadores, una misión no formulada: producir un sujeto para la ciencia a partir de un dispositivo sobre exigente que busca no solamente subsanar o disimular las deficiencias que el sistema educativo nacional tiene desde el nivel básico hasta el superior, sino también lograr que, a pesar de ello, el personaje producido pueda ser capaz de competir en el plano internacional de la producción científica en tanto encarnación de las expectativas de calidad y excelencia propuestas por los objetivos de los programas de formación para la investigación.

II.

¿Cómo llega alguien a ser investigador/a? ¿Se trata de vocación o de destino? ¿Se trata de un proyecto propio o es el resultado de una meta proyectada por otros? La idea de la ciencia como vocación se remonta al mismo nacimiento de las instituciones científicas. Noble (1993) señala que en el origen de las instituciones científicas hay una misma raíz: el interés por la trascendencia y por lo sagrado, lo cual explica la muy antigua asociación entre la vocación religiosa y la vocación científica. Ambas exigen la vida célibe, en tanto que en ambos casos se solicitaba la entrega total de quien era llamado por la vocación y que era lo único que justificaba el sacrificio de los lazos afectivos y familiares considerados distractores y debilitadores de la virilidad necesaria para tan alta empresa. ${ }^{2}$ La Real Academia Española, en su diccionario (RAE, en línea), reconoce cuatro acepciones para el término vocación, que son congruentes con ese nexo entre la vida religiosa y la vida científica: 1) Inspiración con que Dios llama a algún estado, especialmente al de religión; 2) Advocación; 3) Inclinación a cualquier estado, profesión o carrera; y 4) Convocación, llamamiento.

Ciporkin (2004) señala que,

etimológicamente, la palabra vocación proviene de vocatio que indica la acción de llamar y el hecho de ser llamado. En lengua latina tuvo significaciones tales como reflejo del verbo voco, del que deriva llamar,

2 Huelga decir que, a partir de semejante fundamento, no es extraño que el principio simbólico rector de este ámbito haya sido el asociado con la masculinidad, dado que el ámbito doméstico y familiar, considerado el propio de las mujeres y, por lo tanto, femenino, se oponía a los ideales requeridos para la vocación científica. 
hacer venir, convocar. Por influencia del cristianismo alcanza un sentido particular cuyo uso es aplicado en ámbitos religiosos. Desde este punto de vista, vocación es el llamamiento por el cual Dios "inclina a cualquier estado de vida" (Ciporkin, 2004: s/p).

Esta autora habla de que el término vocación se amplió más allá de lo religioso para referirse a: "vocación profesional, vocación artística, científica, etc., a cierta atracción que ejerce un particular objeto del que es difícil sustraerse", y llama la atención sobre una idea subyacente "de ser llamado por Dios para cumplir una determinada misión [lo cual] alude a un sujeto que es elegido y por esta elección estará destinado a una obra que le ha sido asignada". Argumenta que "la vocación como llamado puede situarse en el punto donde un sujeto abrocha y liga el nombre de una profesión o quehacer de la cultura con su quehacer singular".

A principios del siglo XX, Weber distinguió las condiciones exteriores de la vida académica de la "vocación íntima" que, en esa época, estaba determinada por una especialización antes desconocida. Afirma que "sólo la especialización rigurosa da al trabajador científico la conciencia plena de haber conseguido, tal vez por primera y única vez en su vida, algo permanente" (Weber, 2006: 91). Y agrega:

No está hecho para la ciencia el incapaz de ponerse anteojeras y de convencerse a sí mismo de que su destino depende de hacer la conjetura correcta en tal o cual párrafo de tal o cual manuscrito. Nunca sentirá en sí mismo lo que podríamos denominar "vivencia" de la ciencia. Sin esta embriaguez, absurda para los profanos, sin esta pasión, sin la sensación de que "han debido pasar miles de años para que yo apareciese y pusiera a prueba esta hipótesis", no se tiene vocación para la ciencia y hay que dedicarse a otra cosa. Nada es valioso para el hombre si no puede realizarlo con pasión (Weber, 2006: 91).

En nuestra época y en nuestro medio, Pérez Tamayo (1996) discute a la vocación como algo derivado de predisposiciones congénitas. Este autor considera que, aunque el concepto de vocación tiene "bases biológicas reales" -en el sentido de disposiciones genéticas que dotan al individuo de ciertas aptitudes para ciertas tareas-, éstas no son el factor definitivo para la determinación de lo que un individuo logra hacer con sus dotes especiales, ya que el momento histórico más importante es la coincidencia entre dichos elementos biológicos necesarios y la estructura de la sociedad en que ocurren. Este científico afirma que la vocación no existe - "El joven no hace bien lo que le gusta, sino que le gusta lo que hace bien", dice-, y que dicho concepto refleja una idea demasiado esquemática de la realidad, una postura simplista ante el mundo:

En mi opinión, el hombre tiene un repertorio de posibilidades mucho más amplio que el implícito en el estrecho concepto de "vocación", mientras que sus opciones profesionales están rigurosamente limitadas por la estructura de la sociedad y la época en que le toca vivir. Muchos de mis amigos científicos podrían 
fácilmente haber sido otra cosa, pero las circunstancias determinaron que la ciencia le saliera al paso y quizá algún buen profesor tuvo el privilegio de mostrarles, no sólo por su trabajo sino por toda su vida, el atractivo de dedicarse a tal actividad profesional. La prueba de fuego vino cuando hicieron su primer experimento y les salió bien. Estoy seguro de que en ese momento varios o muchos de ellos decidieron que, realmente, la ciencia era su "vocación” (Pérez Tamayo, 1996: s/p).

En cuanto a la consideración del quehacer científico como destino, hay que partir de la definición que se da de este término en el diccionario de la RAE, la cual tiene varias acepciones que agrupamos en dos sentidos principales: 1) fuerza desconocida que se cree obra sobre los hombres y los sucesos. Encadenamiento de los sucesos considerado como necesario y fatal. Hado, fortuna, sino. Circunstancia de serle favorable o adversa esta supuesta manera de ocurrir los sucesos a alguien o a algo. Consignación, señalamiento o aplicación de una cosa o de un lugar para determinado fin. Pero también, 2) empleo (ocupación), lugar o establecimiento en que alguien ejerce su empleo, y meta o punto de llegada.

La combinación de los dos sentidos que se le da al término destino se juega en la discusión sobre lo que produce la decisión de dedicarse a la ciencia, pero va más allá en tanto que habla acerca de la capacidad de elección y las determinantes de ésta, así como de los márgenes de libertad y autonomía que hay para elegir. Esto tiene que ver con el debate teórico acerca de cómo se toman las decisiones e inclusive de si puede llamarse "decisión" a cualquier acción que se emprenda, o qué tanta libertad y responsabilidad individual hay en las acciones que se llevan a cabo (Bauman, 2007). Es decir, podría formularse la pregunta siguiente: ¿cómo intervienen el azar, la suerte, esa fuerza desconocida y fatal, en tener la ocupación de científico como meta vital? Responder que se trata del destino implica tanto dejar de lado los propios intereses y deseos, como desconocer las determinantes que participaron para la elección, muchas de ellas de orden de la estructura histórica y sociocultural, y otras provenientes de un plano subjetivo. Significa también asumirse como carente de capacidad de agencia, es decir, sin la posibilidad de lidiar con dichas determinaciones.

Esta discusión también está involucrada en la comprensión del quehacer científico como un proyecto personal, pero desde un ángulo opuesto, es decir, desde la postura que sostiene que es el sujeto quien es el artífice de sus decisiones y que su meta es planteada por él mismo de manera racional y será alcanzada por un acto de voluntad. Ciporkin (2004) explica que el concepto de "proyecto" es una de las nociones centrales en la obra de dos filósofos del siglo XX que han tenido relevancia en el pensamiento acerca del ser y de la existencia. Señala que fue Heidegger quien primero habló del proyecto como algo central en la historia del pensamiento y lo designa como la posibilidad de comprensión del ser, lo cual es retomado después por Sartre en el marco de la filosofía de la acción, quien propuso que "el hombre se convierte en aquello que él mismo hace". Desde este planteamiento se deriva que todos los actos del individuo son parte de un proyecto fundamental: la manera que se elige de estar en el mundo expresando una elección original en circunstancias parti- 
culares. Por su parte, Simone de Beauvoir planteó también la centralidad de un proyecto personal para las mujeres, como vía para una existencia más allá de la identidad asignada por su papel de otro frente a los varones, es decir, para su liberación.

La idea del proyecto de vida incluye una dimensión temporal determinada que engloba tanto el pasado como el presente y el futuro, unidos por un hilo conductor y orientados a su concreción a través de la voluntad. Por otra parte, habría que pensar que interpretar una vida como un proyecto cumplido -o no- es algo que suele hacerse a posteriori, cuando se hace una relectura del recorrido vital a partir de una óptica que pone en perspectiva al pasado y al presente, en relación con la meta final alcanzada. Esto tiene como efecto la apariencia de que el sujeto tenía conciencia del camino en que "fue puesto", la voluntad para seguirlo y la capacidad de distanciarse un tanto respecto a este para luego analizarlo y comprenderlo. Dice Ciporkin: "Estas conceptualizaciones suponen una cierta libertad humana en cuanto a la posibilidad de desear que eso ocurra, destacando también la intencionalidad y la autonomía del sujeto" (Ciporkin: 2004: s/p).

Por último, asumir que se ha llegado a la formación de investigación como resultado de un proyecto determinado por otros significa dar todo el peso a elementos del contexto como condicionantes ineludibles, entre otros, la organización del sistema escolar, la tradición familiar, la obediencia a un deseo ajeno, etc. Bourdieu señaló en sus trabajos en torno al campo educativo (1997, 2003 y 2008), que la reproducción social tiene lugar a través de la diversidad de los títulos que la escuela concede o deja de conceder, que las modalidades de herencia son históricas y que el peso de la trasmisión de un capital educativo es determinante en el futuro que se modela para uno mismo. Pero también advierte acerca de la usual ceguera que impide reconocer esas determinaciones en el actuar de los propios académicos, en parte como resultado del narcisismo de éstos, que prefieren pensarse como sujetos libres cuyas decisiones son tomadas estrictamente a la luz de la razón (Bourdieu, 2006).

III.

En 2001, Bourdieu (2003) se preguntaba si el oficio científico era un campo como los demás. Señalaba que en este campo ocurría lo mismo que con otros campos, en el sentido de que en éste hay posiciones y representaciones particulares que dan lugar a presiones y estrategias de operación vinculadas con la posición individual y la de los competidores en el ámbito particular del campo y en función de la información disponible y de las específicas estructuras cognitivas. Afirmó que las estrategias individuales para operar en el campo científico y sus posibilidades de éxito dependen de la posición subjetiva ocupada en la estructura que conforma dicho campo, con lo cual hace referencia a las determinaciones estructurales que el campo tiene en los actores, de manera similar a lo que ocurre en otros campos, y de la primacía de dichas determinaciones sobre las capacidades o aprendizajes subjetivos. No obstante, para reflexionar acerca de las particularidades del campo científico, planteó la siguiente pregunta: “¿cuáles son los mecanismos que crean su especificidad y, 
simultáneamente, su irreductibilidad a la historia de lo que allí se engendra?” (Bourdieu, 2003:15). La respuesta que formula este autor se compone de varias partes. Por una parte, afirma que:

La especificidad del campo científico depende, por un lado, del hecho de que la cantidad de historia acumulada es, sin duda, especialmente importante, gracias, sobre todo, a la "conservación" de las adquisiciones de una manera especialmente económica, por ejemplo, con la formación y la formulación, o con las infinitas facetas de un tesoro lentamente acumulado, de gestos calibrados y de actitudes convertidas en hábitos (Bourdieu, 2003: 67-68).

Es decir, el campo científico es el resultado de la acumulación de distintos capitales simbólicos, entre los cuales está uno fundamental: los gestos calibrados y las actitudes convertidas en hábitos. Bourdieu no creía que el quehacer científico fuera algo que se realiza a partir de una conciencia conocedora que actúa, como suele creerse, conforme a ciertas normas explícitas de la lógica y del trabajo experimental, sino que es más bien un "oficio": "es decir, un sentido práctico de los problemas que se van a tratar, unas maneras adecuadas de tratarlos, etcétera" (Bourdieu, 2003: 73). Si bien, dice, el campo científico es, como otros campos, el lugar de prácticas lógicas, tiene la particularidad de que, aunque el habitus científico es también -igual que el deporte o el arte-, una teoría realizada e incorporada, éste tiene la particularidad de que se trata de una inteligencia teórica incorporada en estado práctico. Señala:

La especificidad del "oficio" de científico procede del hecho de que ese aprendizaje es la adquisición de unas estructuras teóricas extremadamente complejas, capaces, por otra parte, de ser formalizadas y formuladas, de manera matemática, especialmente, y que pueden adquirirse de forma acelerada gracias a la formalización... hay que realizar un doble esfuerzo para dominar el saber teóricamente, pero de tal manera que dicho saber pase realmente a las prácticas, en forma de "oficio", de habilidad manual, de "ojo clínico", etcétera, y no se quede en el estado de metadiscurso a propósito de las prácticas (Bourdieu, 2003: 75-76).

Por otra parte, está el papel central que en el campo científico tiene el saber formalizado que se busca dominar, por un lado, a través de la formación y de las formulaciones, y por otro, por los instrumentos (entendidos como la cosificación del saber formalizado). La cuestión de la relación entre la práctica y el método en el campo científico es vista por Bourdieu como el resultado de "un sentido del juego científico que se adquiere mediante la experiencia prolongada del juego escénico con sus regularidades y sus reglas" (2003: 77). Un científico sería definido entonces como "un campo científico hecho hombre, cuyas estructuras cognitivas son homólogas de la estructura del campo y, por ello, se ajustan de manera constante a las expectativas inscritas en el campo" (Bourdieu, 2003: 77). Es decir: habría una relación de determinación estructural entre los elementos propios del campo y el sujeto que ahí se produce. Pero, aclara, dicha determinación sólo es posible porque 
el sujeto mismo se determina a través de un acto de conocimiento y de reconocimiento de las reglas y las regularidades del campo, es decir, en tanto que hay previamente en él cierta disposición que lo hace sensible a las conminaciones implicadas en el campo, con lo que queda entonces ya preparado para responder a ellas "de manera sensata".

Por eso, dice Bourdieu, analizar las disposiciones implícitas en los grupos interdisciplinarios que se constituyen alrededor de un nuevo objeto es una excelente manera de observar y objetivar los esquemas prácticos involucrados en las prácticas científicas. No obstante, advierte, no es sencillo para los sujetos expresar con palabras ni la práctica ni la manera de adquirirla.

\section{IV.}

El proceso de producción del sujeto de la investigación en nuestro país es un enigma si se considera el problemático campo de los estudios de posgrado en este contexto -nivel en el cual se da la formación para la investigación propiamente dicha-. Como afirma Cárdenas (2012),

El posgrado en México tiene problemas. Problemas que lo sumergen en la ola globalizadora, problemas endógenos y problemas que tienen relación con el hecho de que constituye el último eslabón del sistema educativo y de que, por lo tanto, en él se condensan las deficiencias de los eslabones anteriores (Cárdenas, 2012: 87).

¿Cómo es que los alumnos que llegan al posgrado han podido sortear las diversas dificultades y carencias presentes en el sistema educativo mexicano, desde los niveles básicos hasta la educación superior? Parece que esto se debe a dos fenómenos vinculados entre sí; por una parte está la simulación institucional que conduce a insistir en mantener programas de posgrado que no tienen sustento, y por otro, está la confusión entre un "buen alumno" y un investigador. Cárdenas afirma que en los posgrados en México se opera como si en los niveles educativos anteriores se hubiera dado a los alumnos la formación consignada en los planes respectivos y "se pretende fortalecer algo que no tiene cimientos, es la cima de una pirámide carcomida" (Cárdenas, 2012: 92). Y agrega:

La cultura nacional ha ido forjando un esquema del "buen estudiante". Empieza en la primaria y se sigue reproduciendo hasta el postrado... los buenos alumnos quieren coronar sus años de esfuerzo con un posgrado. Ni el sistema ni el buen alumno tienen una idea clara de lo que implica la investigación. El sistema quiere cifras que atestigüen de la voluntad política de promover el desarrollo económico, el posgrado es una buena vitrina. El buen alumno quiere seguir haciendo lo que siempre ha hecho. El interés auténtico por el saber y por la investigación no interviene en este concierto (Cárdenas, 2012: 95).

Por su parte, Porter (2010) afirma la existencia de una brecha entre el sistema escolar mexicano y los programas doctorales en los que se forma a los investigadores, la cual, según este autor, 
tiene que ver con que la mayor parte de dichos programas se han pensado y diseñado a partir de las experiencias de países del primer mundo, esto es, con criterios, sistemas educativos y, por tanto, alumnos, totalmente distintos a los que egresan del sistema educativo mexicano y cuyos niveles de exigencia no corresponden a las circunstancias nacionales. Por esto, dice Porter, entre otras cosas, los niveles de exigencia en los programas de doctorado en México suelen ser desproporcionadamente altos, ya que se quiere competir en el plano internacional con los investigadores formados en el extranjero.

Probablemente por lo anterior es que en los posgrados en México se hace un enorme esfuerzo por producir, a pesar de todo, un sujeto determinado a través del proceso de formarse para la investigación, haciendo abstracción de su procedencia y de los rasgos que muestra su perfil como estudiante. Este esfuerzo se concreta en esmerados procesos de selección, pero sobre todo en el diseño sofisticado de artefactos académicos en donde no sólo se imparten cursos, se ofrecen seminarios, se revisan multitud de textos y obras de diversos autores y se realizan coloquios, sino que incluyen un dispositivo particularmente complejo en el núcleo de esta formación: la dirección individualizada de las investigaciones de tesis por parte de investigadores-profesores. Por otra parte, estos artefactos, más allá de su contenido académico, ${ }^{3}$ están mediados por el diseño de un modelo topográfico articulado y caracterizado por espacios de distintas clases, así como por una temporalidad bien organizada y por puestas en escena artificiales, por composiciones gestuales, trayectorias de ida y de regreso (De Certeau, 2004) y, sobre todo, por la producción de un personaje particular: el investigador científico.

V.

Al hablar de la profesión científica, Weber (2006) afirmó que, en su tiempo, los jóvenes mostraban una tendencia a rendir a ciertos ídolos un culto fundado en el saber común: la "personalidad" y la "experiencia personal". Dice:

Ambas están íntimamente vinculadas y prevalece la idea de que la segunda constituye la primera y es parte esencial de ésta. La gente se esfuerza por "acumular experiencias personales", ya que con esto se promueve una personalidad. Y si no se logra, al menos hay que actuar como si se hubiese recibido ese don. Antes, en alemán, esa "experiencia personal" se denominaba "sensación", y se tenía entonces una idea más correcta del significado de la "personalidad". En el terreno de la ciencia, sin embargo, sólo tiene "personalidad" el que se dedica exclusivamente a su trabajo científico (Weber, 2006: 94)

De esta manera, Weber plantea que en la experiencia se busca forjar un tipo particular de personalidad. Siguiendo esta idea, es posible pensar que la formación para la investigación en nuestros

3 El aspecto de cómo se articulan los programas académicos de los posgrados no será abordado aquí, pero sería importante conocer cuál es la lógica específica que soporta su diseño, a pesar del desfase que hemos mencionado. 
días y en nuestro medio es una experiencia que conduce directamente a promover la personalidad del investigador. ¿Cuál es esa personalidad y en qué consiste la experiencia en la cual se busca forjarla?

En relación con la personalidad, ya hemos dicho en un trabajo anterior (Palomar, 2010) que en el ámbito académico se producen tanto una subjetividad específica como fruto de una cultura particular, determinada estructuralmente por el tipo de capitales simbólicos (prestigio, notoriedad, etc.) que caracterizan el campo académico y científico, y por las formas de poder en juego dentro de dicho campo (Bourdieu, 2008).

¿Quiénes son y cómo son quienes forman el mundo académico? En sus trabajos sobre el mundo académico, Bourdieu señala que estos personajes se caracterizan sobre todo por un narcisismo desmedido que produce la ilusión de autodeterminación. Afirma que, aunque es obvio que las relaciones sociales y las determinaciones estructurales atraviesan el ámbito en el que estos realizan su práctica profesional, los académicos suelen considerarse a sí mismos libres e independientes de toda coerción social, lo cual, señala, se desprende de la exaltación del propio Yo a partir de la creencia de que su profesión los hace pertenecer a una especie superior. Dicho Yo se produce a través de los distintos momentos del proceso de ingreso y promoción dentro del mundo académico, en el paso por su estructura y sus rituales, así como por la repetida realización de las prácticas académicas, todo lo cual va progresivamente estableciendo una selección rigurosa que produce una élite privilegiada. Se trata de un Yo que permitiría hablar de la configuración de un personaje particular determinado tanto por la distribución relacional de sus integrantes de acuerdo a sus orígenes sociales y sus conexiones, sus recursos políticos y económicos, sus trayectorias académicas, sus publicaciones, prácticas profesionales, reconocimientos y posturas políticas, como por la manera en que éste participa en la cultura institucional y en que es afectado por sus diversos circuitos simbólicos. Igualmente, es determinado por el habitus generado a partir de la repetición cotidiana de las prácticas propias del mundo académico, en concordancia con las estructuras mentales que lo tornan un universo autoevidente y familiar en sus determinaciones sociales y en las reglas que ordenan su funcionamiento interno, a todos los niveles. Las mencionadas estructuras mentales incluyen otras funciones, tales como el mismo discernimiento con el cual se realizan los actos clasificatorios y sus productos, con lo cual se elimina la comprensión del discernimiento como acto intelectual consciente o azaroso, ya que se entiende como una de las expresiones de la estructura mental previamente conformada y de la puesta en práctica del habitus (Bourdieu, 2013: 15). De esta manera, la forma de clasificar expresa ya las posiciones diferentes u opuestas implicadas en el espacio social de la academia y la investigación.

En relación con la experiencia de la formación para la investigación, podemos afirmar que ésta se configura básicamente a partir de dos mecanismos señalados por Bourdieu (1997): las estrategias familiares y la lógica de las instituciones educativas. Guichard (1995) ha planteado que la experiencia escolar estimula en los estudiantes un determinado tipo de intenciones sobre su futuro, 
y afirma que esas intenciones pueden convertirse tanto en proyectos como en la ausencia completa de perspectiva temporal acerca de un proyecto. De ello se desprende, según ese autor, que el joven genere un determinado tipo de representaciones de futuro según cómo ha sido la experiencia escolar en cuanto a lo que sabe, lo que aprende y lo que puede hacer.

No obstante, hay que considerar que la experiencia no está en la realidad vacía y esperando a un sujeto previamente existente; más bien planteamos que el sujeto es configurado en la experiencia como efecto del discurso de la ciencia, de la cultura de las instituciones, así como de las prácticas particulares implicadas en la formación de los nuevos investigadores en cada programa. Todos estos elementos entran en juego con los factores del contexto sociocultural del sujeto (Scott, 2001) y en su confluencia producen configuraciones subjetivas particulares que determinan, por ejemplo, el modo en que se establecen vínculos con la cultura académica o en que se elige un particular estilo de vida según el habitus de clase -los modos de comportamiento, el gusto, las actitudes, las formas de ver y sentir-, de género, de edad y del contexto cultural en el que se está inserto. Desde esta perspectiva es posible plantear que la experiencia de formación en la investigación produce un personaje cuya personalidad tendrá rasgos que conjuguen las determinaciones que han intervenido en las historias individuales, las fuerzas del campo académico y científico, y una especie de núcleo identitario profesional común. ${ }^{4}$

De Laurentis (1992) afirma que la experiencia es el resultado de un trabajo hermenéutico vinculado con el proceso de auto representación que define al Yo. A través de dicho proceso, el sujeto interpreta la realidad y se coloca en ésta a sí mismo a partir de nociones que han sido incorporadas de manera inconsciente y que le llevan a posicionarse y a actuar de una particular manera en el mundo. Bauman (2007), por su parte, plantea que la experiencia llega "preenvasada, preinterpretada"; es decir, para este autor la experiencia tiene lugar en el contexto de ciertas creencias del sentido común que hacen inteligible lo vivido según un modo peculiar firmemente instalado previamente. Desde este punto de vista, la formación para la investigación es un ámbito en donde al mismo tiempo que se vive una experiencia predeterminada, se produce un personaje particular cuya personalidad refleja tanto la complejidad del campo académico-científico como las tensiones del contexto socioeconómico y político que lo atraviesan.

VI.

Con este marco conceptual, se tomó la decisión de aprovechar una coyuntura de oportunidad para hacer una sencilla exploración acerca de cómo es el proceso de producción del sujeto de la investigación desde el punto de vista de los alumnos de algunos programas de formación. Dicha

4 La forma identitaria que adoptan los sujetos del mundo académico, dice Dubar, se dirige más a la "realización de sí" y a buscar la "plenitud personal", en concordancia con el proceso de individualización contemporáneo (Beck, 2003) y con los mecanismos meritocráticos destinados a premiar las acciones individuales, dentro de un contexto de gran competencia que ignora las diferencias subjetivas y la incertidumbre propia del quehacer científico, así como la cada vez más frecuente precariedad laboral, a la que se intenta desesperadamente de investir de algún sentido (Dubar, 2002: 148). 
exploración perseguía los siguientes objetivos: 1) comprender qué es lo que lleva a un estudiante a formarse como investigador; 2) conocer cómo es la experiencia de formación en el oficio de la investigación; y 3) obtener un esbozo de cuál es la representación que estos alumnos construyen acerca del oficio y del sujeto de la investigación. ${ }^{5}$

La población para este estudio la conformaron los alumnos de cuatro diferentes programas de posgrado en los cuales se forman investigadores, dos programas de doctorado y dos generaciones de una misma maestría; para establecer la muestra se hizo una selección aleatoria de la lista de asistencia de los grupos, y a los seleccionados se les invitó a participar en la investigación con un mensaje por correo electrónico. Finalmente, la muestra quedó compuesta con aquellos alumnos invitados que aceptaron participar dentro de un lapso determinado, que fueron en total once: cuatro alumnos de doctorado y siete de maestría, de los cuales cinco eran mujeres y seis, hombres. Se les envió entonces un cuestionario por vía electrónica, que estaba compuesto por quince ítems vinculados con cinco categorías analíticas establecidas previamente y que son las siguientes:

1. Motivos involucrados en aprender el oficio de investigador.

2. La experiencia en el aprendizaje del oficio (momentos, eventos, aprendizajes, decisiones, etc.) y juicios acerca del proceso de formación.

3. Definición del oficio de investigar (normas, valores, reglas, hábitos, adjetivos asociados, etc.) y cambios originados en el programa de formación en las ideas previas sobre éste.

4. Descripciones de los profesores y directores de tesis, y de la relación establecida con éstos.

5. El investigador ideal (rasgos, modelos, éxito).

Las respuestas se recibieron también por vía electrónica y posteriormente se procedió a la organización y el procesamiento de los datos, que se analizaron en un primer momento en lo individual; luego se agruparon las respuestas de cada ítem para hacer un análisis transversal de la información. Después se buscó conectar los hallazgos con las categorías analíticas para luego proceder a la interpretación de la información.

VII.

De una manera apretada y en un esfuerzo por extraer algunos puntos a manera de conclusiones de los datos arrojados por el estudio, se intentará ahora una reflexión a partir de las preguntas planteadas en un inicio de este trabajo, y de una discusión de las afirmaciones iniciales.

5 El estudio tuvo límites muy claros y precisos derivados de que, en primer lugar, se contó con bastante poco tiempo para su realización, ya que se hizo intentando explorar algunas hipótesis producidas por el trabajo cotidiano para presentarlas en el seminario sobre el tema que estaba próximo a realizarse. Los alumnos estaban por terminar un ciclo y por salir de vacaciones e incluso en el caso de algunos, por terminar el programa; en ese contexto seguí el impulso de aprovechar la oportunidad asumiendo las implicaciones. No obstante, asumo también que la breve información que se logró conforma un corpus de datos significativos que permite hacer algunas reflexiones y que afina las ideas para futuros trabajos que tengan mayor profundidad. 
Los datos muestran que, para los sujetos encuestados, el destino fue el factor que aparece con mayor claridad como decisivo para estar en un programa de formación para la investigación. Siguiendo a Pérez Tamayo, parece que a estos alumnos la ciencia "le salió al paso" como un camino posible y ellos no hicieron más que internarse por éste. Uno de los sujetos, de hecho, hace referencia clara a "los azares del destino" que intervinieron en el hecho de comenzar un posgrado en investigación. No obstante, otros alumnos hablan de otros intereses relacionados con sus estudios profesionales, tales como buscar soluciones a problemas concretos, querer distanciarse de su ámbito de trabajo, conseguir el grado o lograr especializarse, lo cual, si bien no habla de la elección específica del trabajo científico, sí parece vincularse con una idea de proyecto de vida planteado conscientemente a un plazo más largo. En este punto es importante mencionar la relevancia que ha cobrado la obtención de grados para quienes ya están insertos en el ámbito académico -muchas veces como docentes-, como una forma de asegurar no solamente la permanencia en el trabajo, sino la escalada en los tabuladores laborales que aseguran mejores salarios, además del acceso al sistema de jubilación en mejores condiciones. Si bien ninguno de los alumnos encuestados habló explícitamente sobre este factor, es posible pensar que detrás del deseo de "conseguir el grado" o de "especializarse" -que sí se mencionan-, esté con mayor certeza este elemento que la vocación o un interés genuino en la ciencia. En las referencias a un deseo de "formarse en la investigación", vista como "un área llena de vida y trabajo", que implica "avance, mejora, credibilidad" y que es "muy gratificante", en donde pueden verse quizá algunas señales de dicho interés.

Por otra parte, parece que los sujetos encuestados se piensan a sí mismos como dueños de sus decisiones y capaces de articular un proyecto de vida que, con la ayuda de la voluntad y de algunas virtudes, los llevará a obtener el grado. No se hace ningún intento reflexivo por dar cuenta de los factores que tuvieron peso en la decisión, más allá de la voluntad y de su propio juicio. Por otra parte, la obtención del grado es lo que parece ser la meta planteada al integrarse al programa de formación, más que aprender a hacer investigación.

En cuanto a la manera en que los alumnos encuestados definen el oficio de la investigación, llama la atención lo poco precisas que son las ideas que se tienen en relación con éste, lo cual probablemente tenga que ver con la poca claridad con la cual también el programa explica lo que significa hacer investigación. Por ejemplo, no se obtuvo ninguna mención del interés por el saber o por la indagación sistemática, por comprender o por explicar la realidad. Esto nos hace coincidir con Cárdenas (2012), quien plantea que los alumnos de posgrado parecen funcionar sobre la creencia de que para ser buen investigador es suficiente con ser buen alumno, el cual se define por su sumisión y obediencia al sistema académico, más que por su espíritu cuestionador y crítico, que debería definir a un investigador.

En las respuestas de los sujetos encuestados podemos observar que entre los aprendizajes que se reportan se cuenta la adquisición de construcciones teóricas complejas, aunque ésta sea de forma acelerada y condensada, así como del esfuerzo para lograr que el saber aprendido pase realmente 
a las prácticas en forma de "oficio", con el desarrollo de habilidades metodológicas, técnicas, manuales, del desarrollo de la intuición y del "ojo clínico". No obstante, si se toma en cuenta que el programa de formación para la investigación debe dedicar mucho tiempo a cubrir las deficiencias básicas de la educación de la mayoría de los alumnos (el uso del lenguaje tanto en la expresión oral como en la escritura, la comprensión de textos y el trabajo analítico sobre éstos, el pensamiento lógico, la ampliación del bagaje cultural general, entre otros), así como las restricciones del tiempo y del apoyo económico, es fácil comprender que los aprendizajes mencionados no serán ni lo suficientemente amplios, ni lo suficientemente profundos como para generar un habitus realmente efectivo para una práctica de la investigación como la que debería ser el resultado de los programas de formación.

De entre los distintos espacios y momentos que componen el proceso de formación, los alumnos distinguen dos principalmente: uno, el que se produce con el director/a de tesis; y dos, los coloquios de investigación. Respecto al primero, puede decirse que es un espacio que se ve como el sitio en donde tiene lugar la transmisión de algo más vivencial que racional acerca de la investigación favorecida por la cercanía lograda con el profesor-investigador. En cuanto al segundo, llama la atención que, al mismo tiempo que se describen los coloquios como una experiencia estresante y desagradable, también se dice que "son buenos". Esta manera de referirse a ellos hace pensar en que éstos son un mal necesario; algo que duele, pero forma; el sufrimiento que sin embargo da frutos positivos. Esta manera de vivir los coloquios nos confirma que los vínculos entre los dispositivos de formación científica y de formación religiosa siguen siendo cercanos. Sufrir los coloquios con resignación derivada de la convicción de que son positivos y formativos a pesar de lo desagradables y estresantes que son, hace inevitable recordar el valor que tiene el sufrimiento para los cristianos: se trata de algo que, por una parte, parece estar unido al mal, al pecado (es decir, se merece), pero por otra, es una experiencia muy formativa, ya que, entre otras cosas, el sufrimiento ilumina la conciencia sobre los pecados cometidos, enseña humildad y evita la soberbia. También permite aprender la obediencia y el dominio propio, que purifican y se premian con recompensas.

A partir de la información analizada se puede concluir que los programas de formación de los investigadores operan como artefactos de producción de cierto tipo de subjetividad encarnada en cierto tipo de persona. A partir de esto podrían entonces considerarse a dichos artefactos, siguiendo a Foucault, como "tecnologías del yo", en tanto que la mencionada formación

permite a los individuos efectuar, por cuenta propia o con la ayuda de otros, cierto número de operaciones sobre su cuerpo y su alma, pensamientos, conducta, o cualquier forma de ser, obteniendo así una transformación de sí mismos con el fin de alcanzar cierto estado de felicidad, pureza, sabiduría o inmortalidad (Foucault, 1990: 48).

Se produce un personaje particular a través del dispositivo de la formación para la investigación, el cual está organizado topológicamente y cuyo objetivo entonces no es solamente el apren- 
dizaje académico; dicha formación arranca a partir de un no-lugar -el del lego-, desde donde el aprendiz deberá emprende una trayectoria -la formación-, con un preciso destino final-el examen de grado, que dotará al sujeto de su identidad de investigador-. Esto quiere decir que, además de promover ciertos aprendizajes o un cambio de conducta, de actitud, de estatuto y de modo de vida, dicha formación también conduce a cambios en la autopercepción y en la propia identidad. La formación para la investigación aparece entonces como un proceso producido por un dispositivo secular para formar no solamente el intelecto, sino también los sentimientos y los sentidos, en una especie de ascesis en el camino del conocimiento. ${ }^{6}$ Se trata, en resumen, de aprender ahí a someter la propia vida a un determinado orden y a modelar la propia subjetividad conforme ciertos lineamientos, de tal manera que se adecuen al ideal de la ciencia y, con ello, se tenga un lugar en el orden del conocimiento.

Los programas de formación para la investigación llevan a los aprendices, a través de los discursos y saberes implicados, de su organización topológica y cronotópica, de las formas de hacer (De Certeau, 2000) de los profesores-investigadores y de los directores de tesis, a realizar cierto número de operaciones sobre sí mismos con la promesa de que así lograrán ser el investigador ideal. ¿Cuáles serían esas operaciones y cuál es ese ideal? Entre las operaciones que son necesarias efectuar sobre el sí mismo, pueden mencionarse las siguientes (sin pretender ser exhaustiva): el ordenamiento de los ritmos de la vida cotidiana a partir de las necesidades de formación; la distribución racional de los tiempos que se requieren para atender a otros o las actividades personales; la necesidad de simular los intereses económicos involucrados en la investigación; la delimitación de los espacios destinados al movimiento y los placeres corporales; la formulación de nuevos códigos éticos y de escalas de valores a partir de la prioridad del trabajo académico y de investigación; el acallamiento de las expresiones emocionales o de los aspectos de la vida sentimental o personal, y su subordinación a las necesidades del programa de formación; el desarrollo de una estoica aceptación de la crítica asumida como necesario "elemento formativo" del Yo profesional; el establecimiento de un vínculo personal -"íntimo", dirían algunos- y no muy claro en su naturaleza, con el o la directora de tesis, en donde tiene lugar la transmisión más sutil del oficio de investigar, no nada más como un quehacer sino como un qué ser, es decir, como una subjetividad englobada por una práctica profesional, todo ello imbricado con una dimensión afectiva que puede funcionar tanto como elemento facilitador o como el peor de los obstáculos para la formación del novel investigador, debido a que con frecuencia este elemento se sobrecarga de expectativas y de una intensa transferencia afectiva que nunca se analiza ni se busca disolver.

La formación de los investigadores, pues, no se limita al aprendizaje consciente de contenidos teórico-metodológicos o disciplinares, ni tampoco se agota en la incorporación más o menos advertida de un habitus propio del oficio de investigar. Hemos visto que hay otro nivel importante

$6 \mathrm{Al}$ decir "secular" se pretende distinguir este dispositivo de los dispositivos religiosos que pueden ser muy similares a los de formación para la investigación (tal es el caso de los Ejercicios Espirituales de San Ignacio de Loyola, de los que habla Barthes, 2010), en tanto tecnologías de producción de una precisa subjetividad buscada. 
en dicha formación: la vivencia cotidiana de la cultura académica propia del programa de posgrado creada por el cuerpo de investigadores-profesores en su interacción con los estudiantes y con los pares, y que implica prácticas, discursos, costumbres y rituales comunitarios que generan estructuras de significado particulares, así como la formulación implícita de claves identitarias profesionales y de ciertas formas de hacer que se transmiten tanto a través del actuar como del decir. Por esta vía, los aprendices de investigación son introducidos en un mundo particular de significados, y en un sistema simbólico compartido y compuesto por determinadas formas de vivir y trabajar juntos (Bruner, 2006) estrechamente vinculado con las normas, ideales, valores y bienes simbólicos que se afirman como los propios del mundo académico en el cual se desarrolla. Dicho mundo condensa dos registros principales: el del discurso científico ideal y el de la realidad institucional cotidiana. La confluencia de ambos discursos produce una figura discursiva que es, probablemente, la pieza central en la producción de la configuración subjetiva que se busca lograr a través de los procesos de formación de los nuevos investigadores.

Siguiendo las ideas de Bourdieu respecto a que los poderes propios del sistema de enseñanza no se ejercen si no cuentan con la complicidad activa de quienes los imponen o los padecen, planteamos que la transmisión del ideal de investigador como mecanismo de poder del dispositivo de formación de investigadores, muestra su efectividad al evidenciarse en las clasificaciones que los alumnos aplican a sus profesores o directores de tesis, en tanto que los esquemas con los cuales realizan sus clasificaciones son el resultado de la incorporación de un habitus vinculado al poder que dicho ideal genera para la transformación de la subjetividad de los aprendices. Se trata de una mistificación del quehacer y de sus efectos perversos, dado que la construcción de una imagen glorificada de la investigación y de sí mismo como investigador, es un elemento que lejos de ser formativo, impide concentrarse en la realización correcta de un trabajo (Cárdenas, 2012).

Bourdieu definía al científico como "un campo científico hecho hombre, cuyas estructuras cognitivas son homólogas de la estructura del campo y, por ello, se ajustan de manera constante a las expectativas inscritas en el campo" (Bourdieu, 2003). A partir de la información obtenida en nuestro estudio, es posible concluir que los investigadores que egresan de los posgrados de formación para la investigación muestran que efectivamente son "un campo hecho hombre", pero no necesariamente se trata del campo científico, sino de un campo académico institucional normado por reglas culturales, económicas y políticas específicas. Es decir: si hay una relación de determinación estructural entre los elementos propios del campo y el sujeto que lo habita, el sujeto que se forma en los programas de formación es un personaje cuya disposición lo hace sensible a esas reglas consustanciales al campo del poder determinante. De hecho, podríamos afirmar que las motivaciones para aprender el oficio de investigación que capturamos en nuestro trabajo son las expresiones de la disposición previa a participar en dicho campo y de la sensibilidad ante las conminaciones implicadas en éste que habilitan a los sujetos para ser un "buen alumno", en el sentido de que se sujetará y cumplirá con los requisitos y demandas de la formación, y no un alumno crítico, cuestionador y apasionado por el saber. 
Encontramos que los sujetos encuestados se imaginan a sí mismos ejerciendo el oficio de la investigación estrictamente a partir de lo que conocen: en un avance automático al nivel siguiente de formación, "completando" el trabajo de docencia que ya se realiza, siguiendo las normas institucionales que conocen, trabajando en equipo y con una plaza institucional como investigador/a. En sus previsiones, no obstante, los sujetos vislumbran un futuro optimista que les permitirá llegar a ser lo que el programa les ofrece en términos de formación del carácter y de habilidades, y que podrá desarrollarse en las instituciones conocidas. No hay trazas de que se visualicen dificultades para su inserción laboral o imposibilidades para el ejercicio del oficio, por lo cual tampoco se exploran alternativas nuevas u otros horizontes. Mucho menos se habla de transformar el orden que priva en el campo institucional de la ciencia.

Por último, pudimos ver que los alumnos encuestados asocian el éxito de los investigadores sobre todo a cualidades personales individuales y no a cuestiones relacionadas con la trayectoria, la formación científica o los resultados logrados con su trabajo de investigación. Es decir, el éxito del programa quedará demostrado si se consigue lo que se señala en los objetivos revisados: lograr "recursos humanos" de "alta calidad y excelencia". Si los egresados son así considerados, esto explica la arrogancia y el narcisismo que suelen presentar los investigadores, ya que, como señaló Latapí (2007), la idea de excelencia conlleva la ilusión de perfección. Es lógico preguntarse si entonces lo que se espera del programa de formación es alcanzar la excelencia más que aprender a hacer investigación.

En el contexto contemporáneo en el que cada vez parece ser más relevante el propio Yo, la propia imagen, el propio prestigio y el reconocimiento que se da a la propia persona, es fácil confundir cuál es el centro del oficio de investigación: ¿es el investigador, su personalidad e identidad, o es el trabajo que realiza? ¿No sería mejor pensar al sujeto de la ciencia como una posición en el conjunto de relaciones formales definidas por dicho ámbito y no como un personaje en el escenario de la política de las identidades? Con esa perspectiva, el centro de importancia estaría en lo que se produce al ocupar la posición de la ciencia, no en el personaje que lo ocupa. Sin embargo, en el contexto actual en el cual cada vez se entiende más a la ciencia como una mercancía y una propiedad privada, la personalidad del científico se considera tan relevante, que se cree que es esta personalidad la que imprime valor al trabajo y no lo contrario.

En síntesis, es posible decir que, si bien parece benéfico para la imagen nacional la cada vez más numerosa producción de egresados de los programas de formación para la investigación -ya que genera indicadores que justifican la inversión económica en el sector de la ciencia y la tecnología, además de contribuir a la producción del imaginario de modernidad y desarrollo nacional que se requiere para participar en el concierto de las naciones-, el desfase que hay entre la educación superior mexicana y el nivel requerido para hacer investigación -y que se evidencia de manera dramática en el posgrado-, ocasiona tanto la irracional sobre exigencia en la selección y la formación de los aprendices de investigación, como la indulgencia paradójica de profesores y funcionarios 
con las limitaciones que se enfrentan en las aulas y que se traduce en el enfermizo "no reprobarás" o en el previo "no te fijarás" en lo que pasa, para seguir cumpliendo con la eficiencia terminal que asegure presupuesto, puestos, prestigio y estabilidad. Todo ello, además, contribuye a producir la "evidencia" de un supuesto éxito del sistema educativo nacional y del rendimiento institucional óptimo. El resultado es la producción de cada vez más generaciones, cada vez más numerosas, de investigadores cada vez más jóvenes y, sin embargo, con cada vez menos posibilidades de encontrar espacios donde hacer investigación en los términos en que han sido formados.

\section{Referencias}

Barthes, Roland (2010). Sade, Loyola, Fourier. Madrid: Cátedra.

Bauman, Zygmunt (2007). Libertad. Buenos Aires: Losada.

Beck, Ulrich y Elisabeth Beck-Gernsheim (2003). La individualización. El individualismo institucionalizado y sus consecuencias sociales y políticas. Barcelona: Paidós.

Bourdieu, Pierre (1997). Capital cultural, escuela y espacio social. México: Siglo XXI. (2003). El oficio de científico. Ciencia de la ciencia y reflexividad. Barcelona: Anagrama.

- y J. C. Passeron (2003). Los herederos. Los estudiantes y la cultura. Buenos Aires: Siglo XXI. (2006). Autoanálisis de un sociólogo. Barcelona: Argumentos.

- (2008). Homo academicus. Argentina: Siglo XXI.

— (2013). La nobleza del estado. Buenos Aires: Siglo XXI.

Bruner, Jerome (2006). Actos de significado. Más allá de la revolución cognitiva. Madrid: Alianza editorial.

Cárdenas, Cristina (2012). "El posgrado en el conjunto del sistema educativo mexicano". En: Chavoya Peña, María Luisa y Sonia Reynaga Obregon (coords.). Diversas miradas sobre los posgrados en México. México: Universidad de Guadalajara, pp. 85-106.

Ciporkin, Martha Beatriza (2004). “¿Vocación o destino?”. En: Revista electrónica de psicología política. Año 2, núm. 6, junio, 2004. San Luis, Argentina. Disponible en: http://www.psicopol. unsl.edu.ar/junio04_nota1.htm [Consultado: 06/10/2015]

De Certeau, Michel (2000). La invención de lo cotidiano 1. Artes de hacer. México: Universidad Iberoamericana/ITESO.

_ (2004). "El espacio del deseo". En: Arte y espiritualidad jesuitas. Principio y fundamento. México: Artes de México, núm. 70, pp. 39-47.

De Laurentis, Teresa (1992). Alicia ya no. Feminismo, Semiótica, Cine. Madrid: Cátedra.

Dubar, Claude (2002). La crisis de las identidades. Barcelona: Bellaterra.

Foucault, Michel (1990). Tecnologías del yo y otros textos afines. Barcelona: Paidós/I.C.E.-A.A.B.

Guichard, J. (1995). La escuela y las representaciones de futuro de los adolescentes. Barcelona: Laertes. 
Latapí Sarre, Pablo (2007). Extractos fotocopiados de la Conferencia Magistral pronunciada en ocasión de la recepción del Doctorado Honoris Causa que le otorgó la Universidad Autónoma Metropolitana, el 22 de febrero del 2007, en la Ciudad de México. México.

Noble, David F. (1993). A World Without Women: The Christian Clerical Culture of Western Science. Oxford: Oxford University Press.

Palomar, Cristina (2010). "Género y subjetividad en el mundo académico". En: Chavoya Peña, María Luisa y María Guadalupe Moreno Bayardo (coords.). Temas selectos de la investigación educativa en Jalisco. México: Universidad de Guadalajara.

Pérez Tamayo, Ruy (1996). "La vocación científica". En: Acerca de Minerva. México: Fondo de Cultura Económica. Disponible en: http://bibliotecadigital.ilce.edu.mx/sites/ciencia/volumen $1 /$ ciencia $2 / 40 / \mathrm{htm} / \mathrm{sec} \_29 . \mathrm{html}$

Porter, Luis (2010). "Prólogo". En: Moreno Bayardo, Ma. Guadalupe (2010). Historias de formación para la investigación en doctorados en educación. México: UdeG/Coecytjal/Plaza y Valdés.

Real Academia Española (RAE), Diccionario en línea, en: http://www.rae.es/recursos/diccionarios/drae

Scott, Joan (2001). "Experiencia”. En: La ventana. Revista de estudios de género. Vol. II, núm. 13. Julio, 2001. Guadalajara: Universidad de Guadalajara, pp. 42-74.

Weber, Max (2006). Política y ciencia. Buenos Aires: Leviatán.

Recibido: 17/11/15

Dictaminado: 12/02/16

Corregido: 12/02/16

Aceptado: 12/02/16 